\title{
Knowledge based Medical Decision Support System using Data Mining
}

\author{
Taranath N L, Roopashree H R, Yogeesh A C, Subbaraya C K, Darshan L M
}

\begin{abstract}
Medical Decision Support System
( MDSS )[7] is a system where information of the patient for promising diagnostic and treatment paths are depicted. It can be developed either as the system for Knowledge base or the system for Learning base[2] Knowledge-based systems[3] are the mappings which are0 human - engineered from the best medical practices and patient data recommendations [1]. The mapping is derived by Learning-based systems using various techniques of data mining, machine learning. Knowledge - based and Learning - based systems are integrated to provide a powerful solution to the information challenge in the existence of incomplete facts. This work designs a framework and implements an ontological representation for Integrated Medical Decision Support System which assists Medical Professionals in making clinical decisions for drug prescription. It employs Knowledge base system for drug prescription to the patients. If the available data is incomplete, it uses concept of machine learning0 to produce solution for the given query [5]. It is best suited for different healthcare environment and many different users including physicians, nurses and other staff who serve in medicalfield. The skeleton is query - based which can be adjusted for use with many different interfaces on the user-end including Desktop, Web-based browsers and Mobile applications.
\end{abstract}

Keywords - MDSS, Knowledge - based systems, Learning based systems, Aggregations, SQL, Data Mining

\section{INTRODUCTION}

The purpose of this work is to develop an integrated medical decision support system, which helps to make decisions for medical professionals regarding prescription of medicine. An Integrated MDSS[2] algorithm accepts queries from user for medicine prescription and it uses knowledge base for processing and produces query result as an output. If the available data is not complete, it uses technique of Artificial Intelligence to represent misplaced data and produces better result. In the proposed work, an

Revised Manuscript Received on July 22, 2019.

* Correspondence Author

Taranath N L, Dept. Of CS \& E, AIT,Chikkamagaluru, Karnataka, E-mail : taranath.taras@gmail.com

Roopashree H R, Dept. of CS \& E, GSSITEW, Mysuru, Karnataka

E-Mail : roopashreehr@gsss.edu.in

Yogeesh A C, Dept. of CS \& E, GEC, Kushalnagar, Karnataka

E-Mail : yogeesh13@gmail.com

Subbaraya C K, Registrar, Adichunchanagiri University, BG Nagara, Karnataka E-Mail : subrayack@gmail.com

Darshan L M, Dept. of CS \& E, AIT, Chikkamagaluru, Karnataka E-Mail : darshan.malathesh@gmail.com
Integrated Architecture is designed that integrates both system for knowledge-base and system for learning-based approaches for making medical decisions. It holds the advantage of the concept machine learning,knowledge representation with structures and inference based on logical aspects to provide vigorous, smart system for decision making process. It permits solution to deal with the relation complexities and medical decision interdependencies. It constructs complete explainable responses to queries and robust for critical missing data[1]. It is based on three important information sources from real world : a huge patient history datasets, a registry for interaction with drugs and a set of prescription protocols for medications.

\subsection{Knowledge Based System :}

Knowledge based MDSS[3] can be represented ontologically. Consider $\mathrm{V}$ set of vocabulary of structures and $\mathrm{A}$ axioms about $\mathrm{V}$, which are constructed using a formal language L . Ontology is a sign - system: $\mathrm{O}=\{\mathrm{L}, \mathrm{V}, \mathrm{A}\}$ where the symbols of $\mathrm{V}$ denote categories and relations between categories or between their instances and $\mathrm{L}$ is a formal language associated to a vocabulary $\mathrm{V}$ and used to declare a set of $\mathrm{L}(\mathrm{V})=\mathrm{A}$, which are usually a declarative formulae.

$$
\text { A large patient }
$$

dataset histories, a drug registry for interaction and a group protocols of medical prescription, these are the 3 real world sources of information that it depends on. It has 2 major functions. It provides a barrier for uniting specific information of a query that allows inquiry of information which is distributed in real time data centers such as RDBMS using a plug in design, stores of semantic facts and semi - structured document repositories.

Pieces of atomic sub-queries which are extracted from dividing the semantic query are integrated to form repositories consisting of knowledge. The part or sub - queries are translated into formats specific to each repository by Plugin and then convert it back to a common semantic format by translating it to facilitate direct processing by semantic reasoner. 


\section{Knowledge based Medical Decision Support System using Data Mining}

In addition to knowledge management, a component is required to identify suitable inference rules for making the decision process. A rule of inference defines the various facts in a knowledge base and its relationship and it also permitslogical deduction of additional information from

basic facts. Proposed framework utilizes rules of inference based on Ontological concepts[8] and semantic reasoner is used to process it. It produces the query result.

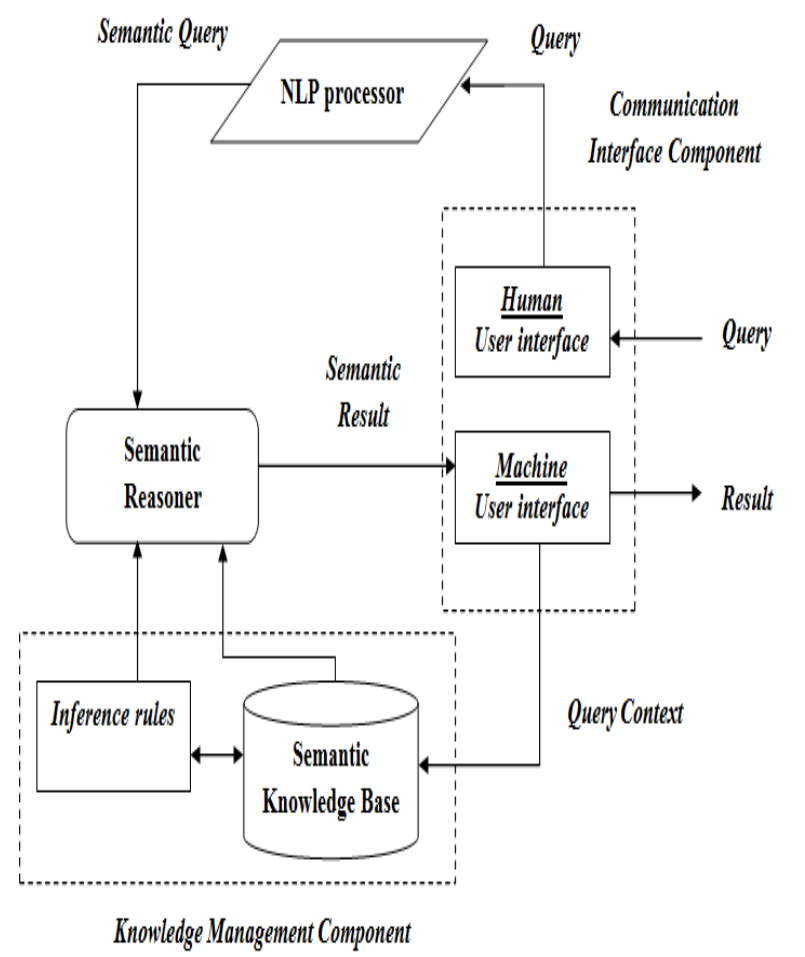

Fig 1 : Architecture of Knowledge based System

\subsection{Motivation for the Proposed System :}

Consistent information interchanging between shared mapping parties under normal circumstances can be obtained using already available methods [4]. At some inauspicious instances such as fact setting and time, where the patient information available is incomplete, the existing techniques seem to fail for practical use. In this work, a framework is designed using ontological concepts, rules of inference and incorpotating the concept of machine learning[7] for interchanging consistent facts between shared different multiple parties.

\subsection{Applications of the Proposed System :}

- It is suitable for many applications of medical healthcare, it helps to represent the missing data even if the information available is incomplete.

- It imparts a solution to understand relationships of medical decisions complexity and inter-dependencies.

- It is compatible with many different interfaces at the user end, which includes mobile applications, desktop applications

and web - based browser applications

- It uses the expert rules [9] of
inference for decision making purpose that helps to get exact results for proper medical decision .

- It is designed in such a way that missing values are predicted directly from the raw data, which is useful in proper medical diagnosis [8].

\section{REVIEW OF LITERATURE :}

\subsection{Rule Based \& Evident Based Systems :}

The system for knowledge base [5] based on Rule Based Fuzzy Logic or Rule Based Systems \& Evidence Based Systems [23]. Fuzzy logic Rule Based [3] is a form of knowledge base and to diagnose disease and pain in patient, it has established several important techniques and mechanisms. Consider an example : Learning Technique using RVM used in patients who are not capable of verbal communication, for management of pain. The technique can be used by the medical staff in measuring pain [15] which is an add-on to Vector machine algorithm using pattern recognition. For enhancement of the productivity of fuzzy set theory [19], a theory is proposed called Rough set theory to accessorize fuzzy set and to deal with indeterminateness and uncertainty . It does not required data like statistical probability distribution, basic assinment of probability and grade of member ship of value of possibility is said to be its main advantage .

The Systems for Rule Base \& Systems for Evident Base [18] are likely to capture the information on area experts and are converted into expressions that can be evaluated as rules. When more number of rules have been integrated into a rule base, the working knowledge will be evaluated against rule base by integrating rules until a final result is obtained.

This system helps in keeping store of a huge volume of facts and processed data. To get the physician doctors and CDSS together, evidence based technique played an important role. It appears to be the most powerful tool to improvise clinical care and patient outcome. It has the ability to increase the quality and safety as well as deducting the cost [ 20 ]. Knowledge base is made up of rules, inference engine integrates rules with the data of the patient and the communication mechanism is helpful in showing outcome to the users and to supply input to the system. In few cases, like chest pain management, the robust guidelines from a knowledge base server is said to be much more productive than others. 


\subsection{Knowledge Based Expert System}
M. Frize
and
R. Walke
[4]

presented

Knowledge - based expert system provide case - based reasoning abilities. The Knowledge - based clinical decision support system consists of rules usually in the form of IF - Then statements . The data is mostly connected with these rules. For example generate a warning if the pain intensity reaches some certain level etc. The knowledge base is generally made of three main parts : Knowledge base, rules of inference and communication mechanism . They modified raw patient data into patient cases and then provided rules of inference to perform search queries which matches very nearly instances[1]. This method was not fruitful and could suffer a notable loss of performance when partial patient data is provided (e.g. patients leave-out details, or access restrictions does not allow to access remote medical records ). Also it is hard for an expert to transform their knowledge into distinct rules [ 1 ].

\subsection{Knowledge based approach for Medical Decision Support Systems}

Knowledge based [5] can be Fuzzy Logic Rule Based or Rule Based Systems \& Evidence Based Systems [25]. Fuzzy Logic Rule Based [3] is a form of knowledge base and has achieved several important techniques and mechanisms to diagnose the disease and pain in patient. For example RVM Learning Technique is used for pain management in patient who cannot communicate verbally. The pattern recognition technique can assist medical staff in measuring the pain [15] which is an extension of Vector machine algorithm. For improving the effectiveness of fuzzy set theory [19], Rough set theory can be proposed to complement fuzzy set and to deal with vagueness and uncertainty. Its main advantage is that it does not need data such as probability distribution in statistics,

basic probability assignment and grade of membership of value of possibility.

Rule Based Systems \& Evidence Based Systems [18] tend to capture the knowledge of domain experts into expressions that can be evaluated as rules. When a large number of rules have been compiled into a rule base, the working knowledge will be evaluated against rule base by combining rules until a conclusion is obtained. It is helpful for storing a large amount of data and information. For closing the gap between the physicians andCDSSs, evidence based appeared to

be a perfect technique. It proves to be a very powerful tool for improving clinical care and also patient outcomes. It has the potential to improve quality and safety as well as reducing the cost [20]. Knowledge base contains the rules, inference engine combines rules with the patient data and the communication mechanism is used to show the result to the users as well as to provide input to the system. In certain case, such as of chest pain management, the adaptive guidelines from

a knowledge base server

prove to

be much more effective than others.

\section{KNOWLEDGE1BASED1SYSTEM}

The Knowledge based MDSS[3] developed using the Protégé tool. The developed model is tested. The parameter considered for Test Case generation includes Name of the Test, Sample Input, Expected Output, Actual Output and Remarks. Various test cases considered here are creating the Patient Instances, creating the Medical condition instances, creating the Drug instances, and creating the Doctor instances, Graphical view of the medical decision support system framework and viewing the query results. The inference rules are designed in accordance withthe patient dataset which defines the semantics of different values for the data attributes, to transform the numerically coded data recordsinto corresponding instances of the Patient class. These rules were then applied to all records to create a semantic knowledge-store of dataset, usinga plugin created specifically for this data. Other patient information (such as medical conditions), was mapped in similar fashion.

\subsection{Design Considerations for Knowledge based MDSS}

- In order to design the Knowledge based MDSS four different classes were considered. They are Doctor, Patient, Medical Condition and Drugs. As listed in Table

- The Patient records were drawn from the Govt. Hospitals of the rural places. Nearly 500 patient instances were considered.

- Suggestions regarding the Medical Condition and Drugs took from 10 Doctors, who are working at the Govt. Hospitals of the Remote areas.

- The Knowledge based MDSS were built for the General Physician Application so nearly 44 Medical Condition related to General Physician treatment were considered. As listed in Table 3.2

- Nearly 200 Drugs which are used to treat for the Medical Conditions mentioned above and almost 100 Drugs which contraindicates with the above mentioned Medical Condition were considered. The Drugs considered here are available at the Remote places of the Govt. Hospitals.

\subsection{Architecture for Knowledge based Medical} Decision Support System

The Knowledge based MDSS[3] can be represented ontologically. Let $\mathrm{V}$ be the set of vocabulary of structures and A axioms about $\mathrm{V}$, which are formulated in formal language $\mathrm{L}$. Ontology is a sign - system : $\mathrm{O}=\{\mathrm{L}, \mathrm{V}, \mathrm{A}\}$ Where the symbols of $\mathrm{V}$ denote categories and relations between categories or between their instances and $\mathrm{L}$ is a formal language associated to a vocabulary $\mathrm{V}$ and used to declare a set of $\mathrm{L}(\mathrm{V})=\mathrm{A}$, which are usually a declarative formulae 


\section{Knowledge based Medical Decision Support System using Data Mining}

A large patient dataset histories, a drug registry for interarction and a group protocols of medical prescription, these are the 3 real world sources of information that it depends on. It has 2 major functions. It provides a barrier for uniting particular information of a query that allows querying of distributed information in real time repositories like RDBMS using a plug in design, stores of semantic knowledge and semi - structured document repositories .

Pieces of atomic sub-queries which are extracted from dividing the sematic query are integrated to form corresponding knowledge stores. The sub-queries are translated into formats specific to each repository by Plugin and then convert it back to a common semantic format by translating it to facilitate direct processing by a semantic reasoner .

In addition to management of knowledge, a component is required to identify suitable inference rules for making the decision process. A rule of inference defines the various facts in a knowledge base and its relationship and it also permits logical deduction of additional information from basic facts. Proposed framework utilizes rules of inference based on Ontological concepts[5] and semantic reasoner is used to process it. It produces the query result.

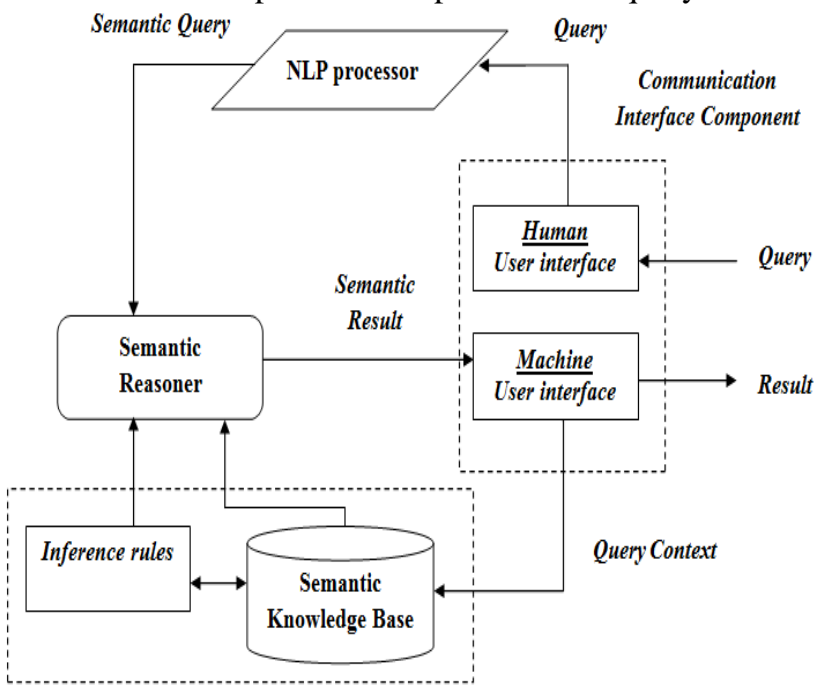

Knowledge Management Component

Figure 2 : Architecture for Knowledge based MDSS

\subsection{Flow chart diagram for Knowledge based}

\section{Systems}

Knowledge base system [3] has 2

major functions . It presents a barrier for uniting particular information of a query that allows querying of distributed data in real time repositories like RDBMS using a plug in design, stores of semantic knowledge and semi-structured document repositories.

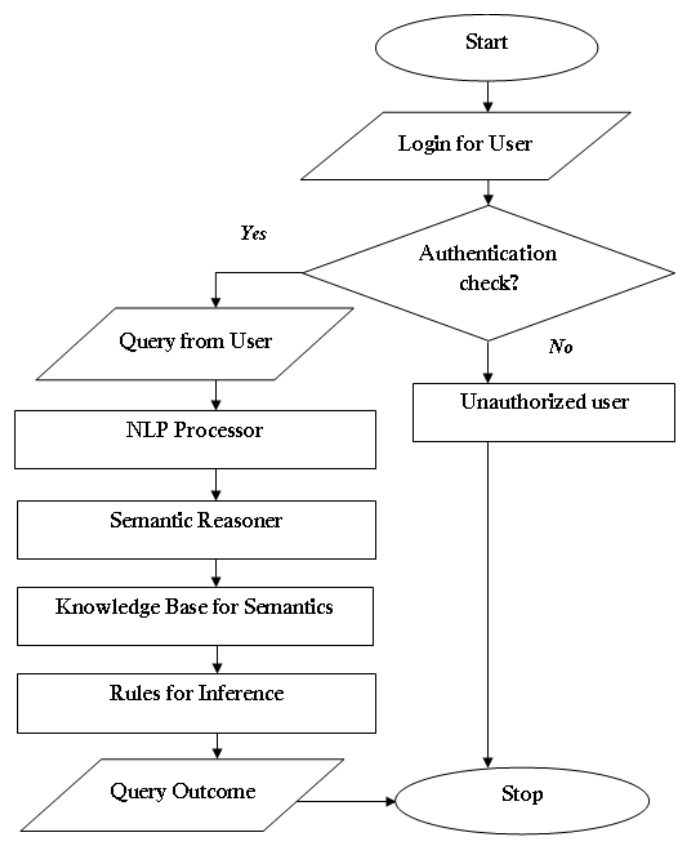

Figure 3 : Flow chart diagram for Knowledge based Systems

3.4 Experimental Results :

3.4.1 Creating the Patient Instances :

From the different hospitals of Government situated at different rural places, nearly 5000 Patient Instances were aqcuired. These instances include various attributes of the patient like Name, ID , Contact Number, Gender, Age , E-Mail ID, Contact Address , Mobile No , Bloodgroup , TreatedBy , Current _ consuming _ drug,

Past_condition , Current_condition ,

Drug_for_current_condition etc ., Fig 4 demonstrates Patient Instances Creation.

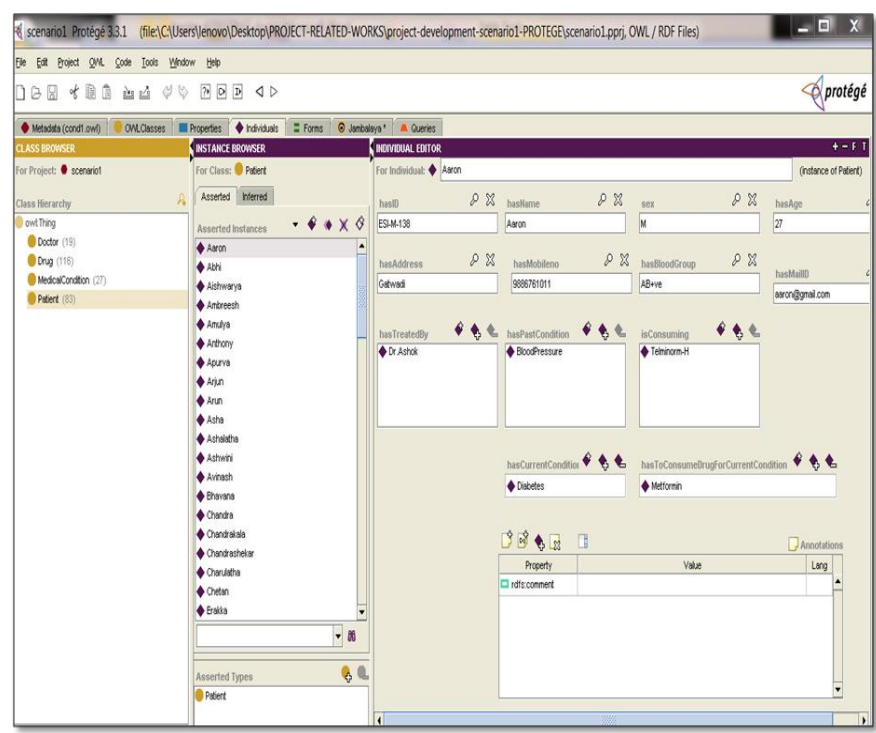

Fig 4 : Creating Patient Instances 


\section{4 .2 Creating Medical Condition Instances *}

Nearly $44 \quad$ Medical Condition

instances related

to General Physician

treatment were considered . Pregnancy , Burns and Scalds, UTI , Gastritis , Chickenpox , Asthma , Migrane, Throat pain, Diabetes, Haematoma ( Blood Clot ), Blood Pressure, etc ., are few of the medical conditions included in these instances. Along with these medical conditions corresponding symptoms $w$ ith each of these medical conditions, its causes and related tests were also created.

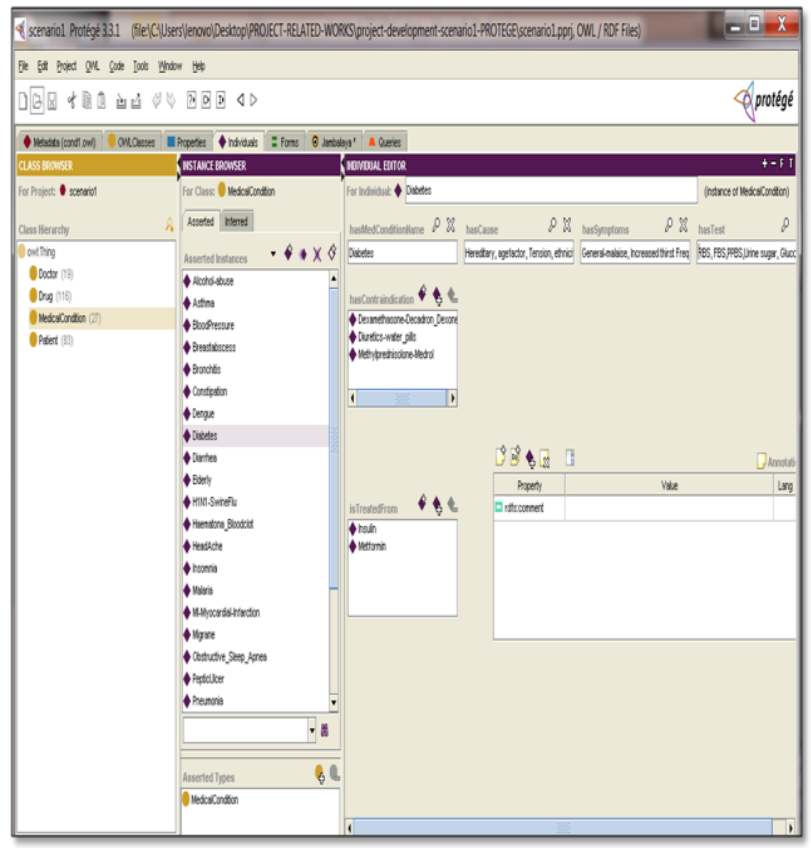

Fig 5 : Creating Medical Condition Instances

\subsubsection{Creating Drug Instances}

Nearly 200 Drug instances which are used to treat for the Medical Conditions mentioned above and almost 100 Drugs which contraindicates with the above mentioned Medical Condition were considered. The Drugs considered here are available at the Remote places of the Govt. Hospitals. These instances include various attributes of the drug like Drug_Name, Code, Company Name, Expiry-date, and Manufacture-date.

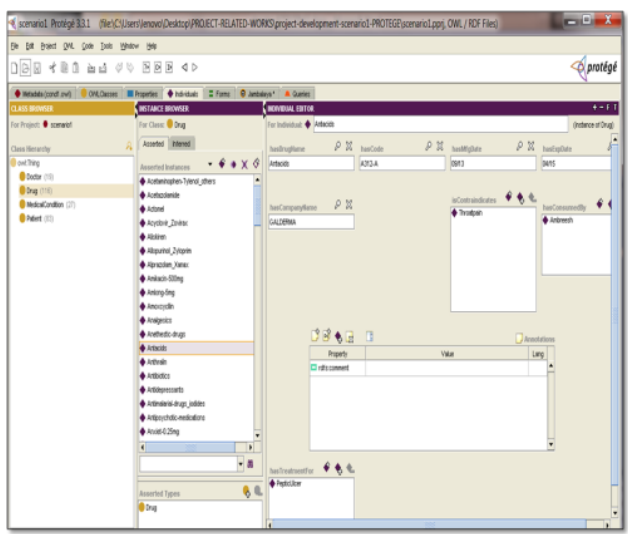

Fig 6 : Creating Drug Instances

\subsubsection{Creating Doctor Instances}

Suggestions regarding the Medical Condition and Drugs took from 100 Doctors, who are working at the Govt. Hospitals of the Remote areas. These instances include various attributes of the Doctor like Doctor Name, Doctor Id, Sex, Age, Address, Mobile no, Mailid, Designation, Qualification and Working Hospital.

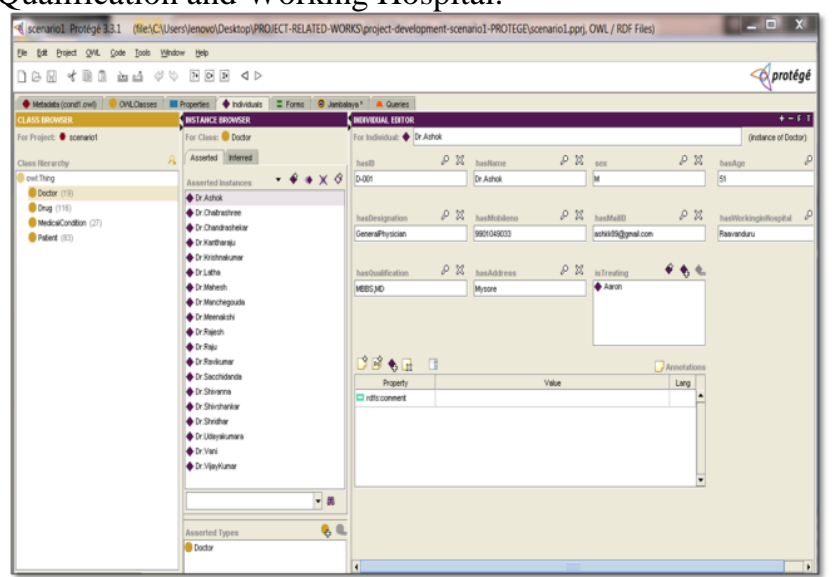

Fig 7 : Creating Doctor Instances

\subsubsection{Pseudo code for designing the attributes related to Patient}

The attributes that relate to only Patient class was identified such as bloodgroup etc. and the end user interface is designed by using the following code snippet.

$<$ ?xml version="1.0" encoding="UTF-8"?>

<tells uri="" xmlns="http://dl.kr.org/dig/2003/02/lang">

$<$ defattribute name="hasBloodGroup"/>

$<$ range string >

$<$ attribute name="hasBloodGroup"/>

$</$ range string $>$

$<$ functional $>$

$<$ attribute name="hasBloodGroup"/>

$</$ functional $>$

$</$ tells $>$

\section{CONCLUSION}

This paper discusses the knowledge based systems and presents an abstraction for aggregating query specific information. It is responsible during decision making process for identifying relevant rules of inference. The response for the user query and the query result is produced by the query execution component.

In this work,

an

Integrated

Medical Decision Support System is implemented to help doctors or medical practitioners, who are working at faraway locations for drug prescription. In this approach, we have integrated System for knowledge base with different algorithms which are extensively used for classification with the concept of machine1learning to1 predict incompletevalues 1bytaking into account 1 of patient indications or symptoms*. 


\section{Knowledge based Medical Decision Support System using Data Mining}

\section{REFERENCES}

1. M.M.Abbasi and S. Kashiyarndi, "Clinical Decision Support Systems: A discussion on different methodologies used in Health Care", International Journal of Computer Science and Information Security, Vol. 8, No. 4, pages 249-256, 2010.

2. M. Frize and R. Walker, "Clinical decision-support systems for intensive care units using case-based reasoning", Medical engineering \& physics, Vol. 22, No.9, pages 671-677, 2006

3. Y. Ye and S. J. Tong, "A Knowledge-Based Variance Management System for Supporting the Implementation of Clinical Pathways", Management and Service Science, IEEE-2009, pages 1-4, 2009.

4. M. Goadrich, L. Oliphant and J. Shavlik, "Learning Ensembles of First-Order Clauses for Recall-Precision Curves: A Case Study in Biomedical Information Extraction", Proc. 14th Int'l Conf. on Inductive Logic Programming, pages 211-214, 2004.

5. T. Mitsumori, M. Murata, Y. Fukuda, K. Doi and H. Doi, "Extracting Protein-Protein Interaction Information from Biomedical Text with SVM ", IEICE Trans. Information and Systems, Vol. E89D, No. 8, pages 2464-2466, 2006.

6. Oana Frunza, Diana Inkpen and Thomas Tran, "A Machine Learning Approach for Identifying Disease-Treatment Relations in Short Texts", IEEE Vol. 23, No. 6, pages 246-246, June 2011.

7. M. Zhu, Z. Zhang, J. Hirdes and P. Stolee, "Using machine learning algorithms to guide rehabilitation planning for home care clients", BMC medical informatics and decision making, Vol. 7, Issue 1, pages 41-43, 2007.

8. D. Rossille, J. Lauren and A. Burgun, "Modeling a decision-support system for oncology using rule-based and case-based reasoning methodologies", International Journal of Medical Information, pages 299-306, 2005

9. Y. Ye and Z. Jiang, "A Semantics Based Clinical Pathway Workflow and Variance Management Framework", Service Operation and Logistica and Informatics, IEEE, pages 758-763, 2008.

10. Avrilia Floratou, Sandeep Tata, and Jignesh M. Patel, Member, IEEE, "Efficient and Accurate Discovery of Patterns in Sequence Data Sets", IEEE Transactions On Knowledge and Data Engineering, Vol. 23, No. 8, pages 30-37 August 2011.

11. P. Patel, E. Keogh, J. Lin and S. Lonardi, "Mining Motifs in Massive Time Series Databases", Proc. of IEEE Int'l Conf. Data Mining (ICDM), pages 370-377, 2002.

12. A. X. Garg, N. K. J. Adhikari and H. McDonald, "Effects of Computerized Clinical Decision Support Systems on Practitioner Performance and Patient Outcomes: A Systematic Review", JAMA, pages 1223-1238, 2005.

13. L. Lin, P. Hu, O. R. Liu Sheng, "A decision support system for lower back pain diagnosis: Uncertainly management and clinical evaluation", Decision Support Systems, pages 1152 -1169, 2006.

14. M. Frize, C. M. Ennett, M. Stevenson and H. Trigg, "Clinical decision support system for intensive care units: using artificial neural networks", Medical Engineering \& Physics, pages 217-225, 2001.

15. D. J. Spiegel halter and R. P. Knill-jones, "Statistical and Knowledge-based Approaches to Clinical Decision-support Systems with an Application in Gastroenterology”, J. R. Statist. Soc., pages 55-77, 2004

16. E. Sivasankar and R. S. Rajesh, "Knowledge Discovery in Medical Datasets Using a Fuzzy Logic rule based Classifier", IEEE International Conference on Electronic Computer Technology, pages 208-213, 2010

\section{AUTHORS PROFILE}

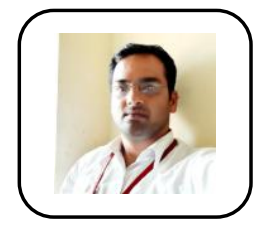

Dr. Taranath N L working as Asst. Professor in AIT, Chikkamagaluru has completed his Ph.D degree from Visweswaraiah Technological University, Belagavi. He has published more than 5 journals and 15 Papers in National/International conferences held at various cities throughout India. His research interests are in the field of Data Mining, Artificial Intelligence, Machine Learning.

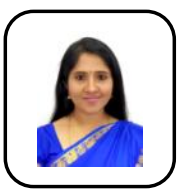

Dr. Roopashree H R working as Asso. Professor in GSSITEW, Mysuru has completed her Ph.D degree from CHRIST University, Bengaluru. He has published more than 5 journals and 15 Papers in National/International conferences held at various cities throughout India. His research interests are in the field of Wireless Sensor Networks, Artificial Intelligence, Machine Learning, Image Processing

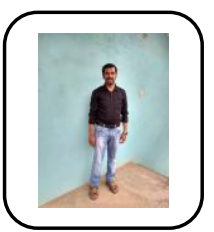

Dr. Yogeesh A C working as Asst. Professor in GEC, Kushalnagar has completed his Ph.D degree from Visweswaraiah Technological University, Belagavi. He has published more than 5 journals and 15 Papers in National/International conferences held at various cities throughout India. His research interests are in the field of Data Mining, Wireless Sensor Networks, Adhoc Networks, Machine Learning.

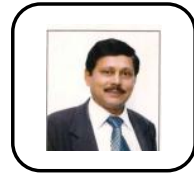

Dr. Subbaraya C K, Registrar, Adichunchanagiri University , BG Nagara has completed his Ph.D degree in Fluid Mechanics from Bengaluru University, Karnataka. He has published more than 50 journals and 100 papers in National and International Conferences. His research interests are in the field of Data Mining,

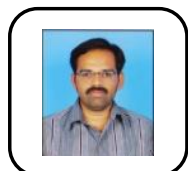
Artificial Intelligence, Machine Learning.

Mr. Darshan L M working as Asst. Professor in AIT, Chikkamagaluru pursuing his $\mathrm{Ph} . \mathrm{D}$ degree in Visweswaraiah Technological University, Belagavi. He has published more than 5 journals and 15 Papers in National/International conferences held at various cities throughout India. His research interests are in the field of Image Processing, Data Mining, Artificial Intelligence, Machine Learning. 